\title{
Math Snacks: Using Animations and Games to Fill the Gaps in Mathematics
}

\author{
Alfred Valdez ${ }^{1, *}$, Karen Trujillo² \& Karin Wiburg ${ }^{2}$ \\ ${ }^{1}$ College of Education, Special Education and Communication Disorders, New Mexico State University, Las Cruces, \\ NM, USA \\ ${ }^{2}$ College of Education, Educational Research and Budgeting, New Mexico State University, Las Cruces, NM, USA \\ *Corresponding author: College of Education, Special Education and Communication Disorders, New Mexico State \\ University, Las Cruces, NM 88003-8001, USA. Tel: 1-575-646-7607. E-mail: valdez1@nmsu.edu
}

Received: September 27, 2013

Accepted: November 14, 2013 Online Published: November 17, 2013

doi:10.5430/jct.v2n2p154

URL: http://dx.doi.org/10.5430/jct.v2n2p154

\begin{abstract}
Math Snacks animations and support materials were developed for use on the web and mobile technologies to teach ratio, proportion, scale factor, and number line concepts using a multi-modal approach. Included in Math Snacks are: Animations which promote the visualization of a concept image; written lessons which provide cognitive complexity for understanding; and active, situated learning activities to facilitate memorable experiences to deepen comprehension. This pilot study compared pre-post test gains for 460 sixth and seventh grade students enrolled in nine different classrooms. In five of the nine classrooms, teachers utilized the Teacher Guide that corresponded with the five Math Snacks animations and one game and in four classrooms teachers used the same Math Snacks animations and one game, but were free to develop their own lessons using available online resources. Results showed moderate and significant pre-post test gains for all six grade students. However, significant gains for seventh grade students were shown only for classrooms where the teacher Guide was used. While it appears that the use of Teacher Guide is useful only for seventh grade, such a conclusion is premature given the small number of classrooms and the exploratory nature of this investigation. Further analyses of moderator variables (e.g., instructional fidelity, learner characteristics) are certainly necessary.
\end{abstract}

Keywords: educational games; mathematics teaching and learning; computer animations

\section{Introduction}

\subsection{The Role of Technology in Teaching}

As laptops and tablets become more accessible to teachers and students in the mathematics classroom, animations and games may play a greater role in instruction. Therefore, it is important to develop effective technological tools that are designed to support student learning and academic achievement. Current technological pedagogy has shown that the effective use of technology, particularly constructivist-oriented technology, may lead to greater student achievement (Wenglinsky, 1998; Schacter, 1999; Lei, 2007). However, technology alone does not necessarily lead to knowledge transfer of mathematics skills and understanding (Devlin, 2011, Gee, 2007; Pea, 1987; Prensky, 2011). Wenglinsky (1998) found evidence that using technology can support mathematics learning but contends that constructivist applications had a more positive effect on math achievement than traditional uses of drill and practice software. Additionally, research has shown that teaching students mathematics conceptually rather than algorithmically leads to a deeper understanding of the content (Resnick, 1983; Kilpatrick, 2001; Ogbuehi, 2007). This deep conceptual understanding of elementary and middle school mathematics content is crucial for students to be successful in high school mathematics and beyond (Kilpatrick, 2001). However, due to the large amount of content covered in 'middle school mathematics' in the U.S. curriculum, it can be difficult to determine which particular concepts students struggle with (Schmidt, Houang, \& Cogan, 2002).

\subsection{Gaps in Mathematics Knowledge}

In 2005, researchers evaluated the scores of standards based assessments for over 24,000 children from five diverse school districts in New Mexico. The analysis showed key gaps in mathematical learning for middle school students 
in the areas of: ratio, proportion, scale factor, fractions, number line, and place value (Wiburg, Korn \& Villa, 2013). Wiburg, et al. triangulated this analysis with teachers' common mathematics misconceptions as shown on the Mathematics Knowledge for Teaching measure, (Hill, Schiling, \& Ball, 2004) and over 800 hours of observation of mathematics classrooms to identify key areas of need for middle-school mathematics instruction. They found that teachers showed knowledge gaps in the same areas (fractions, ratios, number system concepts, etc.).

How can we address key areas of need for middle school mathematics instruction? The Math Snacks grant was written to address these specific learning gaps. The Math Snacks project is a five-year grant funded by the National Science Foundation (award number 0918794) for the development and testing of animations, games, and curricular support materials designed to increase students' understanding of middle school mathematics concepts in the areas of ratio, proportion, scale factor, fractions, number line, and place value. These materials were developed consistent with current theory related to multimedia learning (Mayer, 2002; Moreno, 2006) and constructivist instruction (Greenes, 2009). The materials underwent three years of continuous development which included informal learner observations, student focus groups, and comments from teachers and students.

\subsection{Math Snacks Instruction}

Math Snacks are short animations and mini-games designed to present mathematics utilizing web-based technology. The short animations and games were designed to encourage student learning of mathematics content.

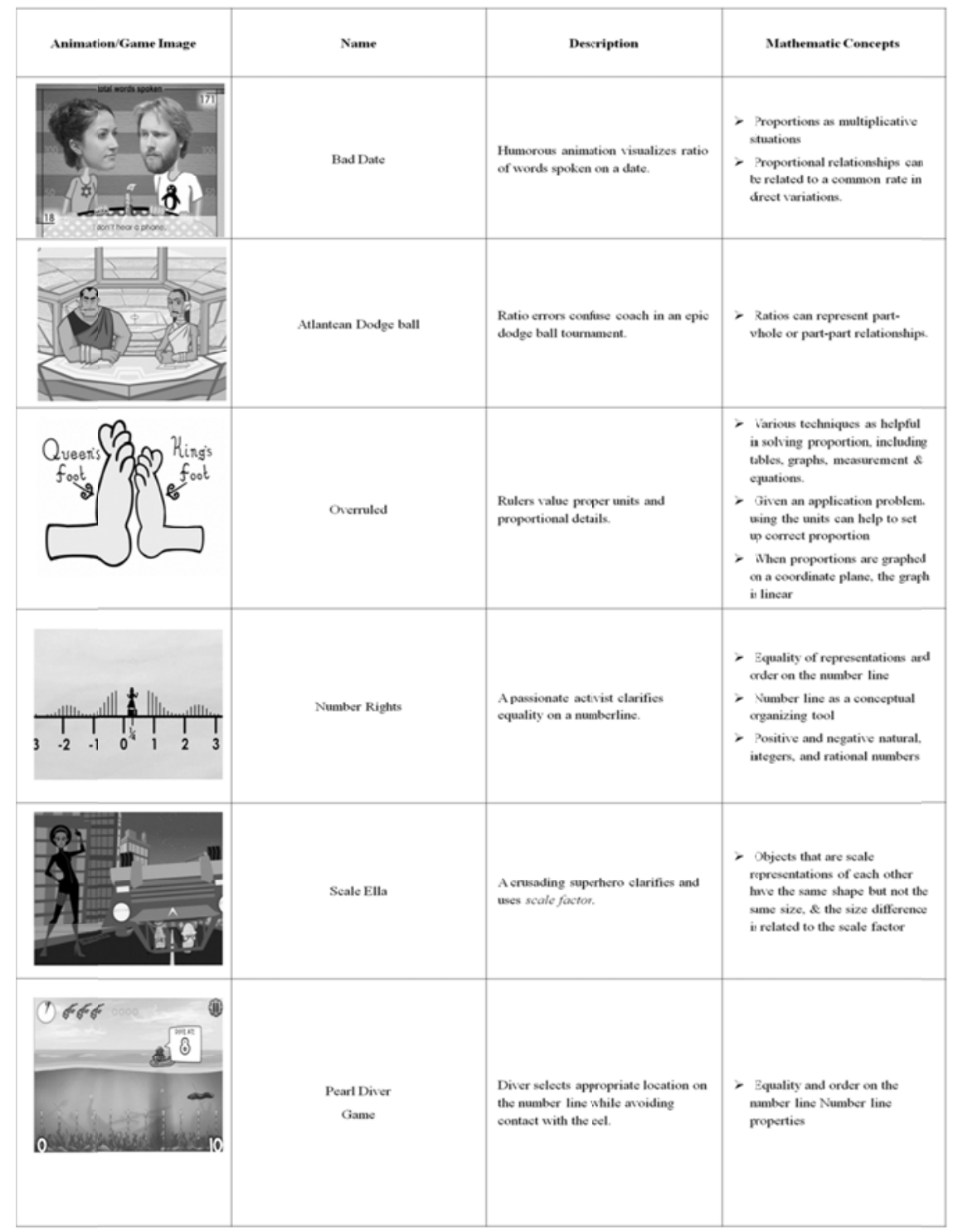

Figure 1: Descriptions of Math Snacks, Five Animations and One Game 
Figure 1 describes the five short animations and one game used in this study. Each Math Snack has a corresponding lesson protocol that is designed to guide the teachers' use of the animations and games. These protocols contain a guided discussion for the animation, an active situated learning activity to be completed after the animation; and suggested questions to facilitate deeper comprehension of the lesson. The lesson protocols as well as the learner guides, teacher guides, example lesson videos and other support materials are available online at http://mathsnacks.com/teachingBD.html

\subsection{Purpose}

One of the goals of the Math Snack Grant was to determine the effectiveness of the Math Snacks curriculum on mathematics achievement. To accomplish this goal, the grant will conduct a large-scale randomized controlled efficacy study in fall 2013 and spring 2014. In preparation for the large-scale study, the Math Snacks research team conducted this pilot study in order to achieve the following objectives:

- Determine the psychometric properties of the Measure of Mathematics Learning.

- Determine the short-term effects of the Math Snacks games and animations

- Determine whether the use of the corresponding teacher guides add to the overall effects of the Math Snacks games and animations.

- Collect qualitative data describing teachers' classroom implementation of the math snacks materials.

\subsection{Research Questions}

- Research Question One: Will students who were taught using the Math Snacks games and animations show growth in mathematical knowledge in the targeted areas of math achievement (i.e., number-line \& ratio/ proportion)?

- Research Question Two: Is student math achievement influenced by teacher's use of the teacher guides that were developed by the Math Snacks grant to support math instruction?

\section{Method}

\subsection{Participants}

Teacher participants were recruited from one urban and one rural southwestern border-school district. Nine teachers from two school districts ( 7 urban \& 2 rural) volunteered to participate in this study. Teachers were given the Math Snacks instructional materials (games, animations, and teacher guides) as well as minimal monetary incentives for their participation in the study. Student participants included 460 students (236 male, 224 female). Some posttest participant attrition occurred for twenty-six sixth grade students (18 control group students, 8 experimental group students) but there was no posttest attrition for seventh grade students. This study was approved by the respective school districts and by the New Mexico State University Institutional Review Board. Parental permission was obtained for all student participants.

\subsection{Procedure}

Students received instruction for eight weeks. In order to examine the effects use of the corresponding teacher guides, five of the nine teachers were asked to follow Math Snacks Teacher Guide (experimental group) and the remaining four teachers were encouraged to develop their own lessons (self-constructed lesson or control group). Classrooms were systematically assigned to each condition. The Math Measure was administered prior to and then following the 8 -week instructional period. Teachers in both groups were asked to integrate five animations and one game during the 8-week period. The researchers observed each classroom on five occasions during the 8-week instructional period. Student learning was operationalized as pre-post test gain score. To investigate whether student learning differed across the two conditions (experimental and control), teachers were systematically assigned to each condition.

\subsection{Reliability and Validly of the Measure of Mathematics Learning}

While student mathematics performance is measured annually using a standardized measure, the purpose of this investigation was to determine more discrete change in mathematics knowledge over time. Therefore, the Math Snacks research team constructed a measure, the Measure of Mathematics Learning, which was intended to measure short-term progress in discrete areas of mathematical knowledge (number line and ratio/proportion).

\subsubsection{Score Reliability of the Measure of Mathematics Learning}

Score reliability was determined using coefficient alpha. Alpha was calculated for the pre-test sample at .88 for 6th 
grade $(n=138)$ and at .83 for 7 th grade $(n=150)$ students. Alpha values above .80 are considered good and values above .90 are considered excellent (George \& Mallory, 2003; Nunnally \& Bernstein, 1994).

\subsubsection{Score Validity of the Measure of Mathematics Learning}

The twenty-seven item measure was constructed from released items that were publically available from national test databases. Three members of the research team located released mathematics test-items from a variety of sources that included the National Assessment of Educational Progress (NAEP), the Massachusetts Comprehensive Assessment System (MCAS), the Florida Comprehensive Assessment Test (FCAT) and the California Standards Test (CST).

Table 1: Measure of Mathematics Learning Test Blueprint

\begin{tabular}{lccc}
\hline & Multiple-choice & Open- ended & Total \\
\hline Number Line & $1,2,3,4,5$ & 5a,6a,6b,6c,6oe & 10 \\
Ratio/Proportion & $7,8,10,11,12,13$, & 9a,9aexp,9b,9bexp, & 17 \\
Total & $14,16,17,18$ & $15 \mathrm{a}, 15 \mathrm{aoe}, 15 \mathrm{~b}$ & \\
\hline
\end{tabular}

The test blueprint for the Measure of Mathematics Learning is shown in Table 1. In order to assure appropriate coverage for grades six and seven, the test items were selected from test banks that were intended for grades five through eight. As Table 1 shows, the items included recognition (i.e., multiple-choice), as well as inference and explanation (i.e., open-ended) items. Twenty-seven items were selected. Ten items pertaining to number line concepts (5 multiple-choice, 5 open-ended) and seventeen items pertaining to ratio/proportion concepts (10 multiple-choice, 7 open-ended) were selected.

\subsection{Qualitative Observations}

Classroom observations were conducted to determine how the classroom teachers carried out their mathematics lessons. Each teacher was observed three times by trained observers who judged how well teachers carried out six key instructional elements. Observers were trained to assign a summary categorical judgment (yes/sometimes/no) to estimate how often each of the six key instructional elements occurred during a mathematics lesson. If the observers determined that the key element was present over $75 \%$ of the time, the categorical judgment was considered as "YES". If the observers determined that the action took place between $50 \%$ and $74 \%$ of the time, the categorical judgment was considered as "SOME". If the observer determined that the action took place less than $50 \%$ of the time, the categorical judgment was considered as” NO”. The key instructional elements included:

- Animation: The teachers' use of the animation to support the lesson.

- Learner Guide Use: The teachers' utilization of the written learner guide (separate support document from the Teacher Guide) to support his/her lesson.

- Activity: How frequently the teacher encouraged an active learning experience where students could gain a deeper understanding of the content.

- Student Engagement: How often students appeared attentive and engaged during the lesson (i.e., asking questions, discussing the content with their classmates, etc.).

- Effective Questioning: How frequently the teacher asked "open-ended” questions that encouraged student inquiry in the lesson rather than "closed-ended" questions that simply requested factual information.

- Effective Classroom Discourse: How frequently the teacher engaged in classroom discourse that encouraged students to interact with the technology, with the lesson, with each other (pertinent to the lesson), or with the teacher for the purpose of learning the lesson content.

The lead researcher reviewed the transcripts of the trained observers and compiled a summary that described how each teacher applied the six key instructional elements. The lead researcher met with the trained observers weekly to recalibrate observational judgments. 


\section{Results}

\subsection{Descriptive Analyses}

Sixth and seventh grade test scores were aggregated by grade level for both the control and experimental groups.

Table 2: Means, Standard Deviations, and Sample Size for Sixth and Seventh Grade Experimental and Control Groups

\begin{tabular}{ccccc}
\hline & & Control & & \multicolumn{2}{c}{ Experimental } \\
Grade Level & Pretest & Posttest & Pretest & Posttest \\
\hline Sixth & 10.46 & 12.93 & 10.73 & 13.21 \\
& $(5.48)$ & $(5.69)$ & $(5.20)$ & $(5.62)$ \\
& $\mathrm{n}=146$ & $\mathrm{n}=146$ & $\mathrm{n}=189$ & $\mathrm{n}=189$ \\
\hline Seventh & 14.27 & 15.12 & 12.50 & 14.74 \\
& $(5.30)$ & $(6.03)$ & $(4.97)$ & $(6.24)$ \\
& $\mathrm{n}=67$ & $\mathrm{n}=67$ & $\mathrm{n}=58$ & $\mathrm{n}=58$ \\
\hline
\end{tabular}

As Table 2 shows, both control and experimental groups showed pre/posttest gains on the Measure of Mathematics Leaning. While the mean pre/posttest gain for sixth grade appeared comparable for control and experimental groups (mean difference $=2.47$ and 2.48 respectively), the pre/posttest gain for the seventh grade appeared much greater for the experimental group in comparison to the control group (mean difference $=2.24$ and .85 respectively).

\subsection{Pre-Treatment Equivalency}

As Table 2 shows, pretest scores were comparable between experimental and control for both sixth and seventh grade. Follow up independent t-tests showed no significant differences in pretest scores between control and experimental groups, $t(359)=.14, p=.89$, and $t(123)=1.92, p=.06$, for sixth and seventh grades respectively suggesting that the groups were equivalent on the dependent measure prior to the intervention.

\subsection{Research Questions}

3.3.1 Research Question One: Will students who were taught using the Math Snacks games and animations show growth in mathematical knowledge in the targeted areas of math achievement (i.e., number-line \&ratio/ proportion)

3.3.2 Research Question Two: Is student math achievement influenced by teacher's use of the Teacher Guides that were developed by Math Snacks grant to support math instruction.

\subsection{Statistics and Data Analysis}

To investigate both research questions, a mixed two-way repeated measures analysis of variance (ANOVA) was conducted with group (experimental \& control) as the between-subject factor and time (pre/posttest) as the within-subject factor. Alpha was set at .05 for all analyses.

For sixth grade students, there was a significant main effect for time, $F(1,333)=131.39, p<.001$, partial $\eta^{2}=.28$. However, the main effect for group and the group by time interaction was not significant. This finding suggests that both the experimental and control groups made significant and parallel gains over time in learning the targeted mathematics concepts. For seventh grade students, there was a significant group by time interaction $F(1,123)=4.09$, $p=.045$, partial $\eta^{2}=.03$. Therefore, interpretations of the main effects were set aside and the nature of the interaction was further investigated with follow-up dependent t-tests. The dependent t-test was not significant for the control group, $t(66)=1.86, p=.067$. However, the experimental group showed a large and significant gain on the dependent measure over time, $t(57)=4.33, p<.001, d=.81$.

In summary, it appeared that sixth grade students made large and significant gains in mathematics knowledge over the course of the intervention regardless of whether teachers used the Math Snacks Teacher Guide or not. However, for seventh grade students, significant and large gains were only shown for those students whose teacher used the Teacher Guide.

\subsection{Treatment Fidelity}

One of the stated purposes of this study was to collect qualitative data describing teachers' classroom implementation of the math snacks materials. 
Table 3: Classroom Observation Summaries

\begin{tabular}{|c|c|c|c|c|c|c|}
\hline \multicolumn{7}{|c|}{ Key Elements } \\
\hline $\begin{array}{l}\text { Teacher } \\
\text { (grade) }\end{array}$ & Animation & $\begin{array}{c}\text { Learner } \\
\text { Guide } \\
\text { Use }\end{array}$ & Activity & $\begin{array}{c}\text { Student } \\
\text { Engagement }\end{array}$ & $\begin{array}{c}\text { Effective } \\
\text { Questioning }\end{array}$ & $\begin{array}{c}\text { Effective } \\
\text { Classroom } \\
\text { Discourse }\end{array}$ \\
\hline \multicolumn{7}{|c|}{ Control Group (Teachers did not use corresponding Math SnacksTeacher Guide) } \\
\hline B $\left(6^{\text {th }}\right)$ & Yes & Yes & Some & Some & No & No \\
\hline$D\left(6^{\text {th }}\right)$ & Yes & Yes & Yes & Yes & Yes & Yes \\
\hline$F\left(6^{\text {th }}\right)$ & Yes & Yes & Yes & Yes & Yes & Yes \\
\hline$G\left(7^{\text {th }}\right)$ & Yes & Yes & Some & No & No & No \\
\hline \multicolumn{7}{|c|}{ Experimental Group (Teachers did use corresponding Math SnacksTeacher Guide) } \\
\hline$A\left(6^{\text {th }}\right)$ & Yes & Yes & Yes & Yes & Yes & Yes \\
\hline$C\left(6^{\text {th }}\right)$ & Yes & Yes & Yes & Yes & Yes & Yes \\
\hline$E\left(6^{\text {th }}\right)$ & Yes & Yes & Yes & Yes & Yes & Yes \\
\hline$H\left(6^{\text {th }}\right)$ & Yes & Yes & Yes & Yes & Yes & Yes \\
\hline I $\left(7^{\text {th }}\right)$ & Yes & Yes & Yes & Yes & Yes & Yes \\
\hline
\end{tabular}

Note: Amount of time Key Element was present - Yes $>75 \%$, Some $50-74 \%$, No $<50 \%$

Table 3 summarizes the qualitative observational data for each classroom. As Table 3 shows, all teachers in the experimental group consistently demonstrated all six of the key instructional elements while teaching the mathematics lesson. However, in the control group, two teachers (one sixth and one seventh grade) were less consistent at demonstrating all of the key instructional elements during their classroom instruction. Teacher B (sixth grade) used the animation and the learner guide, but did not carry out the activities for all of the lessons. This teacher also struggled to maintain student engagement and did not show any evidence of effective questioning strategies or effective classroom discourse. Teacher $\mathrm{G}$ (seventh grade) had similar issues and also did not carry out all of the recommended activities. This teacher also struggled with classroom management. While students appeared engaged during the animation, they quickly lost interest in any further instruction and showed little engagement during the suggested learner guide activities. Subsequently, this teacher did not show evidence of effective questioning or classroom discourse.

\section{Discussion}

Instructional innovations offer the promise of improving instructional effectiveness but do not guarantee a beneficial instructional outcome. As Moreno (2005, p. 14) pointed out, "any technological innovation is fraught with promises and challenges". It therefore becomes critical to carefully investigate the strengths and weaknesses of any instructional innovation. Math Snacks animations, games and curricular materials were developed to address key gaps in mathematical learning. The purpose of this investigation was to develop and pilot the dependent measure (Measure of Mathematics Learning - number line ratio \& proportion), to determine if the Math Snacks materials effectively address certain key areas (e.g., number line and proportion) and to explore whether teachers benefitted from the "extra level of guidance" offered when using the Math SnacksTeacher Guides.

The 27-item Measure of Mathematics Learning showed good internal structure reliability and reasonable face validity. The success of this measure was largely due to the careful selection of test items from nationally recognized test banks, the utilization of a test blueprint in order to assure adequate construct coverage, and the review and modification of the test items from the five-member research team. This measure was used to determine student progress in the targeted mathematics areas (number line \& proportion/ratio).

In terms of program effectiveness, pre-post test scores on the dependent measure showed mixed results. Sixth grade students, regardless of whether teachers used the Teacher Guide, showed large and significant improvement on the dependent measure. However, significant improvement on the dependent measure was only shown for seventh grade students whose teacher used the Teacher Guide. On the surface, it appears that the use of the Math Snacks materials does result in significant growth in the targeted mathematics areas (i.e., ratio and proportion). In addition, for seventh grade, the use of the Math Snacks Teacher Guide appeared to result in a more favorable learning outcome. It could be that the Teacher Guide offerred an added level of guidance that enabled the teacher to more effectively structure and carryout the mathematics lesson. An alternate explanation however, is that the difference in seventh grade outcomes was due to teacher characteristics. In any case, this finding points out the necessity to include classroom 
observational data in any study that attempts to explore the efficacy of an untested instructional program.

In addition, further investigation should study the complex relationship between game-play and mathematical knowledge acquisition. As Gee (2011) points out, our current task is to present logical and testable hypotheses in order to move our understanding of the benefits, limitations, and possible harmful effects of game-based instruction. Further areas of inquiry should include learning context. Both Fe (2008) and Plass et al. (2011) found an increase in affective variables depending upon whether students engaged in individual, cooperative, or competitive game play. However, the question remains whether this increase in affect results in a corresponding increase in mathematical knowledge.

Given the exploratory nature of this pilot study certain limitations should be considered. Due to the small number of classrooms that were included in this study, random selection to condition would not likely have achieved pre-treatment group equivalency. While treatment and control groups showed equivalent scores on the pretest, they may have differed in other areas. Future studies should include a larger sample of classrooms and use either matching or random assignment in order to assure pre-treatment group equivalency. In addition, these findings are limited to sixth and seventh-grade students receiving an eight-week focused instruction in the area of number line and ratio/proportion. These findings may not generalize to other grade levels or other mathematics topics.

\section{Acknowledgements}

This material is based upon work supported, in part, by the National Science Foundation (NSF) grant, award number 0918794, Math Snacks. Any opinions, findings, and conclusions or recommendations expressed in this material are those of the authors and do not necessarily reflect the views of the National Science Foundation.

\section{References}

Devlin, K. (2011). Chapter 17: Bringing Cool into School. Yearbook (National Council of Teachers of Mathematics), 73, 241-249.

Fe, F. (2008). Alternative goal structures for computer game-based learning. Computer-Supported Collaborative Learning, 3, 429-445.

Gee, J. P. (2007). Good video games + good learning. New York: Peter Lang Publishing

Gee, J. P. (2011). Reflections on empirical evidence on games and learning. In Tobias, S. and Fletcher, J.D., (Eds.), Computer games and instruction (pp. 251-278). State University of New York, Albany, NY, USA.

George, D., \& Mallory, P. (2003). SPSS for Windows step by step: A simple guide and reference. 11.0 update (4th ed.). Boston: Allyn \& Bacon.

Greenes, C. (2009). Mathematics learning and knowing: A cognitive process. Journal of Education, 189, 55-64.

Hill, H. C., Schilling, S. G., \& Ball, D. L. (2004). Developing measures of teachers' mathematics knowledge for teaching. Elementary School Journal, 105, 11-30. http://dx.doi.org/10.1086/428763

Kilpatrick, J. etal. (2001). Adding it up: Helping children learn mathematics. ISBN-10: 0-309-06995-5

Lei, J., \& Zhao, Y (2007). Technology uses and student achievement: A longitudinal study. Computers and Education, 49, 284-296. http://dx.doi.org/10.1016/j.compedu.2005.06.013

Mayer, R. E. (2002). Cognitive theory and the design of multimedia instruction: An example of the two-way street between cognition and instruction. New Directions for Teaching \& Learning, 89, 55-71. http://dx.doi.org/10.1002/tl.47

Moreno, R. (2005). Instructional technology: Promise and pitfalls. In L. PytlikZillig, M. Bodvarsson, \& R. Bruning (Eds.), Technology-based education: Bringing researchers and practitioners together (pp. 1-19). Greenwich, CT: Information Age Publishing.

Moreno, R. (2006). Learning in high-tech and multimedia environments. Current Directions in Psychological Science, 15(2), 63-67. http://dx.doi.org/10.1111/j.0963-7214.2006.00408.x

Nunnally, J. C., \& Bernstein, I. H. (1994). Psychometric Theory (3rd ed.). New York: Mc Graw-Hill.

Ogbuehi, P.I., \& Fraser, B.J. (2007). Learning environment, attitudes and conceptual development associated with innovative strategies in middle-school mathematics. Learning Environments Research, 10, 101-114. 
http://dx.doi.org/10.1007/s10984-007-9026-z

Pea, R.D. (1987). Cognitive technologies for mathematics education. Cognitive Science and Mathematics Education. A.H. Schoenfeld ed., Hillsdale, N.J.: Lawrence Erlbaum Assoc., 1987, pp 89-122.

Plass, J. L., O’Keefe, P. A., Homer, B. D., Case, J., Hayward, E.O., Stein, M., \& Perlin, K., (2013). The impact of individual, competitive, and collaborative mathematics game play on learning, performance, and motivation. Journal of Educational Psychology. Advance online publication. http://dx.doi.org/10.1037/a0032688

Prensky, M. (2011). Comments on research comparing games to other instructional methods. In Tobias, S. and Fletcher, J.D., (Eds.), Computer games and instruction (pp. 251-278). State University of New York, Albany, NY, USA.

Resnick, L. B. (1983). Mathematics and science learning: A new conception. Science, $220,477-478$. http://dx.doi.org/10.1126/science.220.4596.477

Schacter, J., \& Milken Exchange on Education Technology, S. A. (1999). The impact of education technology on student achievement: What the most current research has to say. Retrieved from ERIC database (ED430537)

Schmidt, W., Houang, R., \& Cogan, L. (2002). A coherent curriculum: The case of mathematics. American Educator, 26(2), 10-26, 47.

Wenglinsky, H. (1998). Does It Compute? The Relationship between Educational Technology and Student Achievement in Mathematics. Policy Information Center, Mail Stop 04-R, Educational Testing Service, Rosedale Road, Princeton, NJ 08541-0001

Wiburg, K., Korn, K., \& Villa, J. (2013) Understanding Conceptual Gaps in Mathematics: Implications for Educational Policy. Unpublished manuscript. Educational Research and Budgeting, College of Education, New Mexico State University. 